\section{A physical approach to model occlusions in the retinal microvasculature}

O Fleck and T Savin

\begin{abstract}
Blood occlusions in the retinal microvasculature contribute to the pathology of many disease states within the eye. These events can cause haemorrhaging and retinal detachment, leading to a loss of vision in the affected patient. Here, we present a physical approach to characterising the collective cell dynamics leading to plug formation, through the use of a bespoke microfluidic device, and through the derivation of a probabilistic model. Our microfluidic device is based on a filtration design that can tune the particle volume fraction of a flowing suspension within a conduit, with sizes similar to arterioles. This allows us to control and reproduce an occlusive event. The formation of the occlusion can be examined through the extracted motion of particles within the channel, which enables the assessment of individual and collective particle dynamics in the time leading to the clogging event. In particular, we observe that at the onset of the occlusion, particles form an arch bridging the channel walls. The data presented here inform the development of our mathematical model, which captures the essential factors promoting occlusions, and notably highlights the central role of adhesion in these processes. Both the physical and probabilistic models rely on significant approximations, and future investigation will seek to assess these approximations, including the deformability and complex flow profiles of the blood constituents. However, we anticipate that the general mechanisms of occlusion may be elucidated from these simple models. As microvascular flows in the eye can now be measured in vivo and non-invasively with single cell resolution, our model will also be compared to the pathophysiological characteristics of the human microcirculation. Eye (2018) 32, 189-194; doi:10.1038/eye.2017.270; published online 12 January 2018
\end{abstract}

\section{Introduction}

The microcirculation permeates all organs throughout the body to deliver the essential chemicals and metabolites required for continued healthy cellular function. ${ }^{1}$ The capillaries, arterioles, and venules that comprise the microcirculation represent a reticulated terminal network, which also regulates pressure, flow, and temperature of the blood within tissues. ${ }^{2}$ Hence, it is an important component of the vasculature, with a central role in the physiological functions of the body, and any dysfunction of the microcirculation can have severe consequences.

The eye offers a unique window to observe the health and disease of the microcirculation, to perform associated diagnostics, or assess the effectiveness of a treatment. ${ }^{3}$ The retinal microvascular bed is almost two-dimensional on the retina, with a thickness of approximately $200 \mu \mathrm{m}$, and non-invasive measurements of its geometry, topology, and perfusion maps can now be performed rapidly. ${ }^{4,5}$ Most recent in vivo techniques indeed provide a wealth of information on disease and their progression. In sickle cell disease, for example, abnormal erythrocytes stiffen in oxygen-depleted conditions and can occlude the microvasculature. ${ }^{6}$ The associated proliferative sickle retinopathy may cause irreversible damage to the retina, with occlusions occurring in the retinal microvasculature giving rise to vitreous haemorrhage in about $44 \%$ of patients, and may lead to retinal detachment if left untreated. ${ }^{7,8}$ Scanning light ophthalmoscopy has recently been used to quantify the decreased density of perfused microvessels in sickle cell retinopathy (Figure 1), and such approaches are indeed showing great promise for medical applications. ${ }^{9}$

In vivo observations of pathological retinal microcirculation provide an exclusive opportunity to understand the physical mechanism by which the microvasculature is
Department of Engineering, Cambridge University, Cambridge, UK

Correspondence:

T Savin, Department of Engineering, Cambridge University, Trumpington Street, Cambridge CP2 1PZ, UK

Tel: +44 1223332 762;

Fax: +44 1223339263. E-mail: t.savin@eng.cam.ac. uk

Received: 27 October 2017 Accepted: 27 October 2017 Published online:

12 January 2018 
a
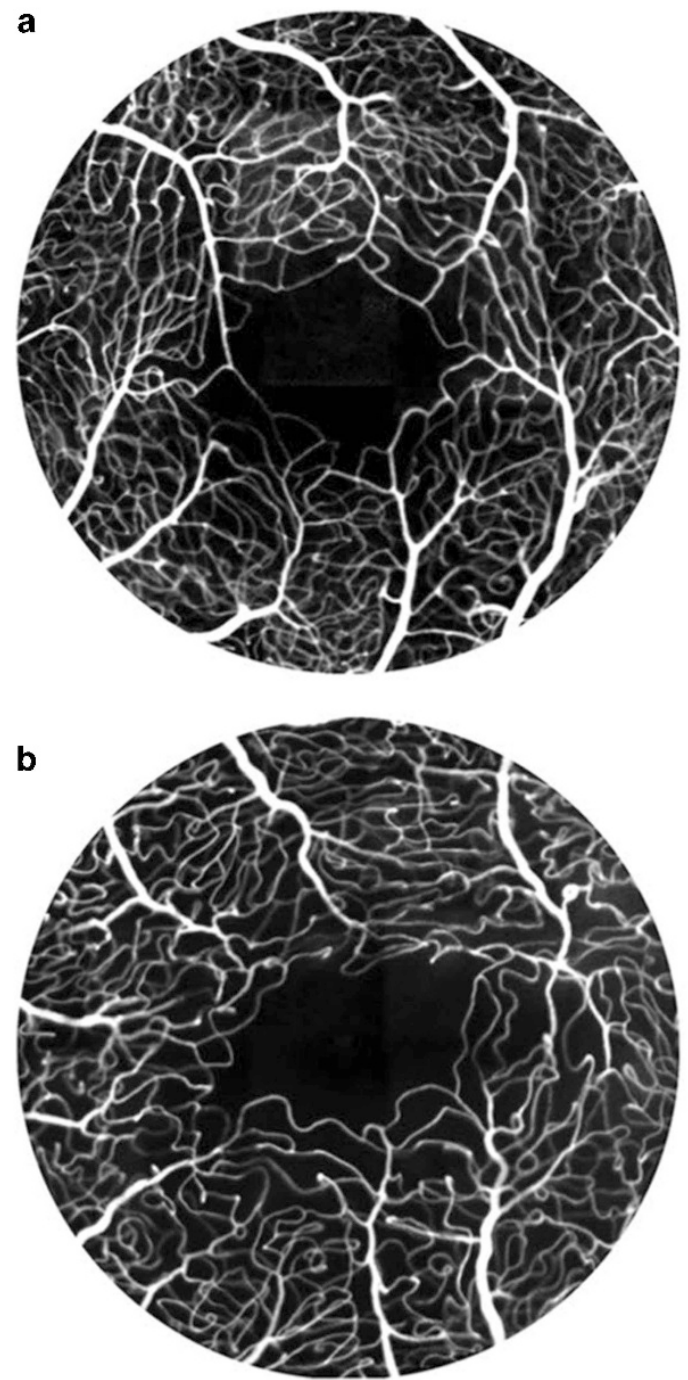
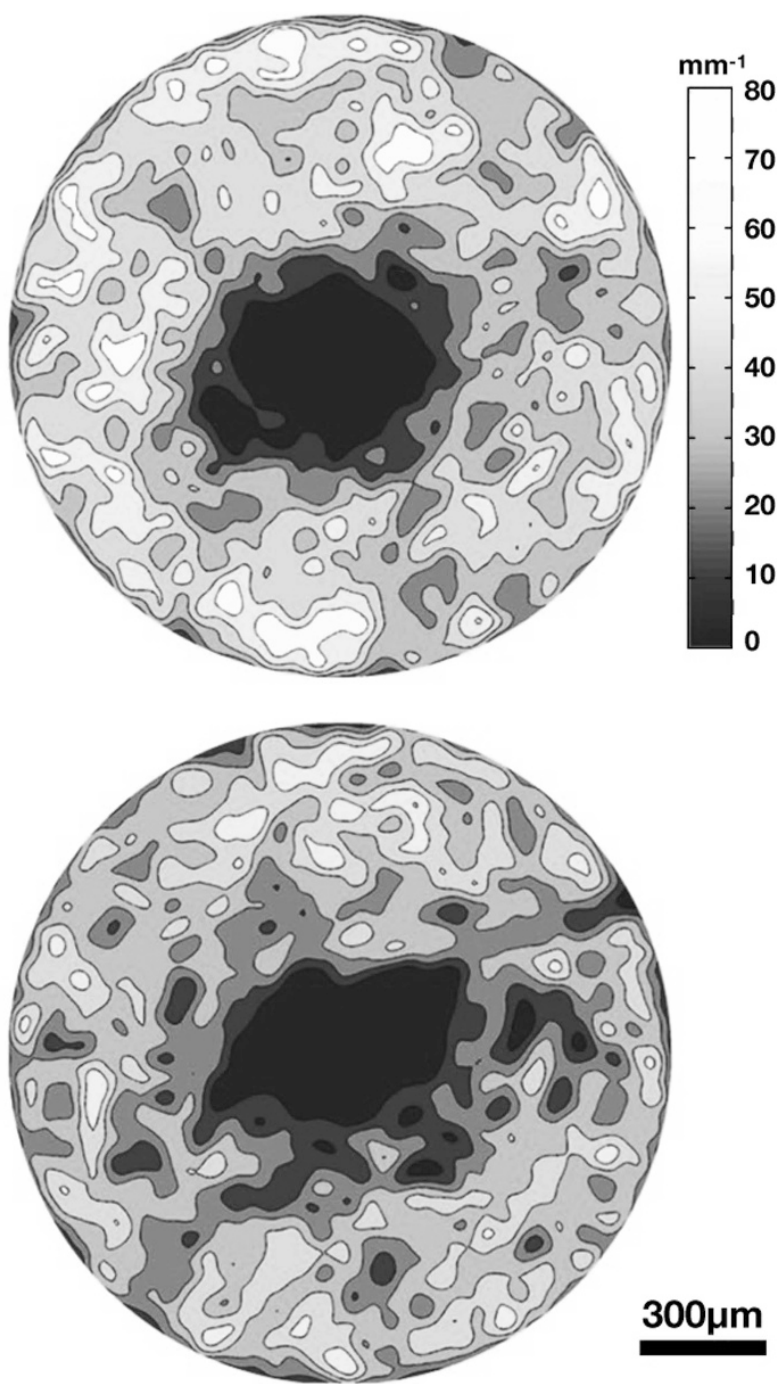

Figure 1 Fluorescein angiographs, obtained by adaptive optics scanning light ophthalmoscopy, show the perfusion maps (left column), which are analysed to calculate the perfused vessel density contours (right column; in perfused length per unit area). (a) is from a healthy retina and (b) from a sickle cell disease patient. The affected retinal vasculature displays greater tortuosity and a decreased perfusion when compared with healthy patients. Reproduced from Chui et al ${ }^{9}$ (@ BioMed Central).

occluded in diseases affecting the blood cells' deformability (observed in sickle cell disease, but also malaria, diabetes, and leukaemia). Isolating the biophysical context of a vaso-occlusion in these diseases can indeed evaluate the distinctive role of cellular interaction, cell stiffness and shape, or adhesion in arresting the blood flow. Following a bottom-up approach, one may then elucidate the dynamics of propagation of these clogs throughout the entire microvascular network, in relationship to their geometrical and topological characteristics. Establishing the hierarchy of these various factors may subsequently help targeting biomedical studies accordingly..$^{10}$ In this paper, we report on a preliminary physical model of microcirculatory flow, which allows measurements of the collective cellular dynamics leading to an occlusion in a microvascular vessel.

\section{Microfluidics: a model for the microcirculation}

The dimensions of the human microvascular network typically range from $200 \mu \mathrm{m}$ for arterioles and venules, to approximately $3 \mu \mathrm{m}$ for capillaries. ${ }^{11}$ Understanding blood flow at these scales has been the subject of numerous studies, ${ }^{1}$ and among the recent experimental approaches, microfluidics has been the science of choice. ${ }^{12}$ Microfluidic devices are microfabricated patterns of submillimetric conduits which can manipulate, and indeed be used to study fluid flow at the micron scale. Microfluidic chips are simple to manufacture (see the section 
'Experimental Design'), allowing fast prototyping, and have numerous applications in bioengineering and biomedical research ${ }^{13}$ (eg, lab-on-a-chip). As shown in recent studies, they appear uniquely suited to investigating and characterising occlusive events. ${ }^{14,15}$ Hence, the occlusion of a capillary by a single cell has been measured and modelled using micron-sized channels, ${ }^{16}$ and microfluidic models of sickle cell blood flow occlusion and rescue have also been reported in larger conduits, ${ }^{17,18}$ with sizes similar to the diameter of arterioles. The latter study was unable, however, to isolate the mechanism of the occlusions.

An assessment of the collective dynamics of blood cells forming an occlusion is indeed lacking. Erythrocytes have an average diameter of $8 \mu \mathrm{m}$, and leukocytes are typically larger at around $10 \mu \mathrm{m}$ in diameter. At the microvascular scale, the use of continuum fluid dynamics to describe blood flow is significantly challenged, ${ }^{19}$ due to the similarity of length scales between the constituent particles and the tube diameter they flow through. In that case, the particulate nature of blood cannot be ignored, and the multicellular dynamics must be assessed at the single cell level to understand flow and its occlusion.

As a starting point, here we simply model the blood as a suspension of microspheres, and we aim at measuring the collective event that initiates an occlusion in a channel of width $~ 3-10$ times larger than the particles.

\section{Experimental design}

We have designed a microfluidics chip consisting of a central conduit, mimicking an arteriole or a venule, through which a monodisperse suspension of particles, emulating the blood, flows as driven by a pressure difference between the inlet and the outlet. The liquid supernatant is drained out of the main channel via small side channels, through which the particles cannot fit. The design principle is shown in Figure 2a, and the actual schematic of the channel in Figure $2 b$. The height of the main channel is designed to be slightly larger than the microsphere diameter, so that the particles flow in two dimensions. The small side channels, whose height is a fraction of the particle diameter, have no physiological equivalent, but through alterations of pressure variables, the concentration of the particles may be tuned along the central conduit by the filtration process these side channels permit (see Figure 2c). The length scales are chosen such that the flow is laminar, at the low Reynolds numbers found in the microcirculation. The goal of this design is to control the formation of an occlusion by adjusting the concentration of particles near their closepack configuration (for which the volume fraction is $\phi^{*} \approx 50 \%$ ). The relevance of these measurements for actual
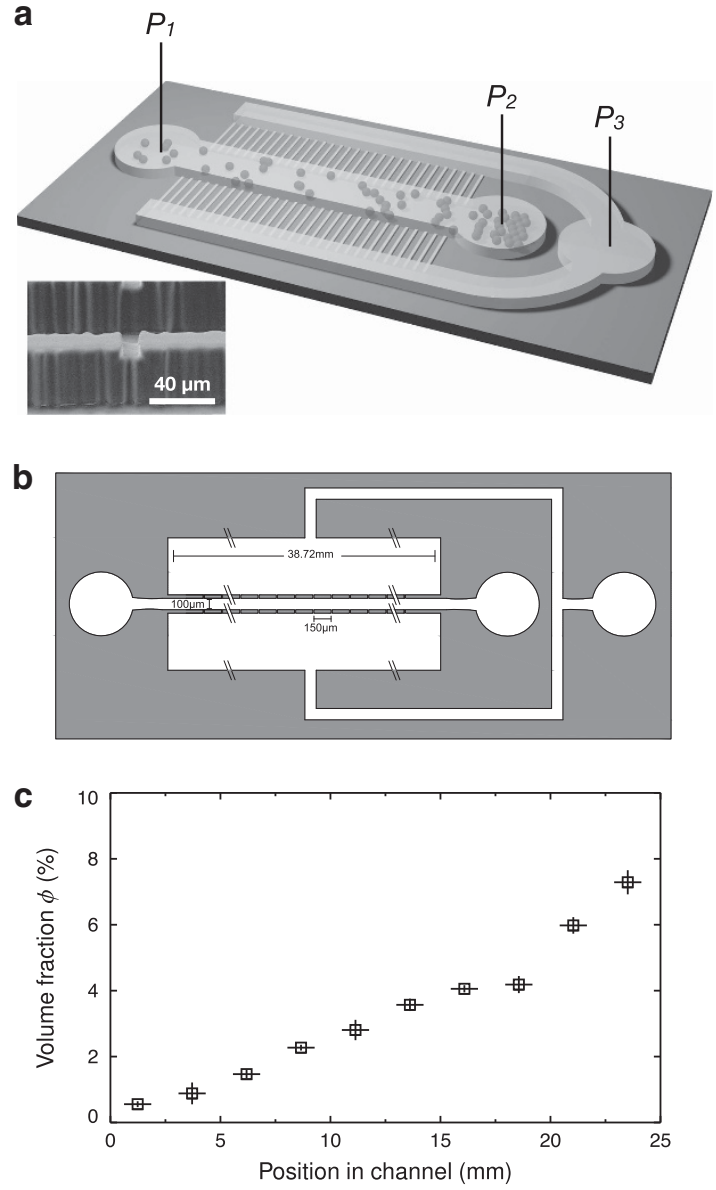

Figure 2 (a) A three-dimensional rendering of the proposed microchannel design, where fluid is drained from the main centre conduit by side channels; the flowing suspension is driven by the difference between the pressures $P_{1}$ (inlet) and $P_{2}$ (outlet), and the filtration is controlled by the pressure $P_{3}<P_{2}<P_{1}$; such design permits the controlled increase in particle volume fraction as the suspension flows. The inset is a scanning electron micrograph showing the opening of the side channel on the wall. (b) A scaled schematic of the microchannel design, where the width of the main channel is $100 \mu \mathrm{m}$ and its height $32 \mu \mathrm{m}$; the smaller side channels have a rectangular cross section of $10 \times 5 \mu \mathrm{m}$. (c) A demonstrated increase in particle volume fraction as the colloid suspension travels through the main channel; the volume fraction is extracted from micrographs acquired at different locations in the channel under steady flow.

blood micro-occlusion is critically discussed later in the text.

The fabrication of the microfluidic chip follows a standard soft lithography procedure. There, a highresolution mask of the channel design is generated with AutoCAD software. It is then used to create a silicon master wafer through contact photolithography, where a curable resin, that solidifies under UV exposure through the mask, forms the design pattern in relief on the wafer substrate. A transparent silicon gel 

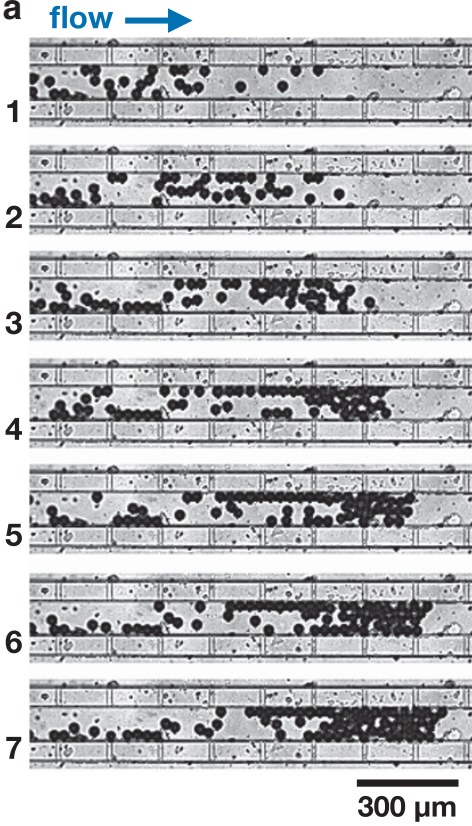

b
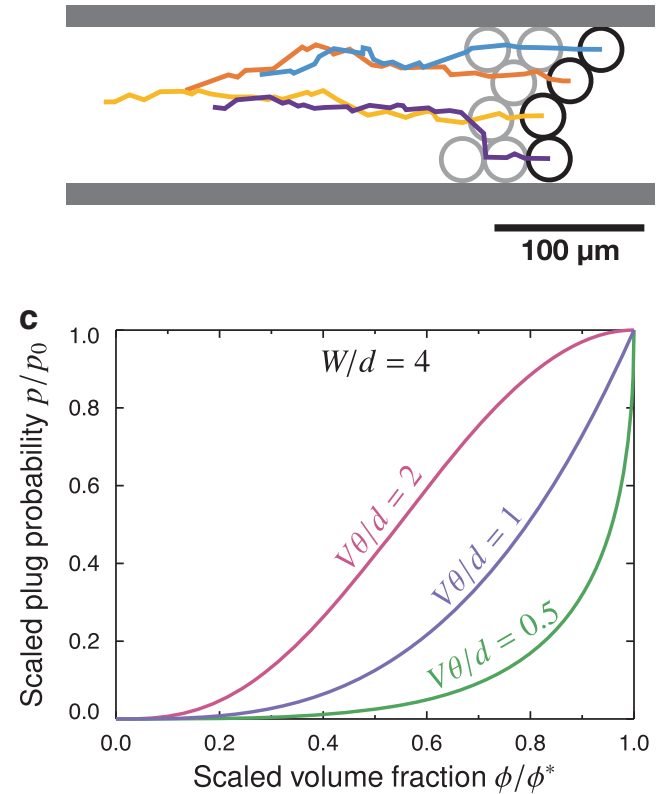

Figure 3 (a) The formation of an occlusion within the microfluidic channel (the images displayed are captured at intervals of $200 \mathrm{~ms}$ ); the formation of an arch, a geometry seen in granular jamming events, can be observed in images 6 and 7, and prevents the passage of particles upstream. (b) Paths of the four particles leading to the occlusive arch; these particles can be observed to flow in the middle of the channel before moving laterally at the onset of the arch's formation. (c) Probability of an occlusion as a function of the suspension's volume fraction, as modelled by Equation (1); the width is set to $W=4 d$ as it is the case in (a) and (b).

(polydimethlysiloxane, PDMS) is then poured over the master wafer and cured. Access ports to the channels are created on the resulting moulded PDMS gel, before it is bound to a PDMS-coated glass slide using a plasma treatment. Tubing is connected to the access ports to perfuse the channels with $30 \mu \mathrm{m}$ microspheres suspension. The height of the central channel is $32 \mu \mathrm{m}$ and the height of the side channel is $5 \mu \mathrm{m}$ (see inset of Figure 2a). The microfluidic chip is mounted onto an inverted video microscope, and the pressures at the inlet and outlet of the main channel, as well as the pressure driving the supernatant through the smaller side channels, are adjusted via hydrostatic reservoirs. The movies can later be analysed via image processing. Typical images of the flowing particles are shown in Figure 3a.

\section{Preliminary results}

Figure 2c shows the volume fraction of particles as measured by counting the microspheres on snapshots of the central channel flow (similar to those shown in Figure 3a) acquired at successive locations. The result shows that, with our filtration design, the concentration of particles in the suspension can be significantly increased (by approximately tenfold here) as it flows along the channel. We show in Figure 3a a series of images capturing the formation of an occlusion in the central channel when a sufficiently high volume fraction is reached. On these images, the smaller side channels can be distinguished as grooves on the top and bottom walls. As the spheres flow near the side walls, they slow down and perturb the flow of other incoming particles. Collective dynamics follow, whereby four particles form an arch bridging the two side walls, blocking particles upstream from passing and forcing them to accumulate behind. Such events have been observed in the jamming of granular flow. ${ }^{20}$ This process is at the origin of the resulting clog, and we believe that similar processes are initiating the vaso-occlusions of the retinal microcirculation seen in sickle cell anaemia and other diseases. The adhesive properties between particles and between the particles and the wall are central to the flow arrest (see the section 'Probabilistic Model').

A more precise investigation of the multi-particle dynamics of clogging can be achieved by tracking the four particles that form the arch. In Figure $3 b$, we show the resulting trajectories as obtained by post-processing the movie of Figure 3a. The extracted motions show some lateral displacements due to crowding, as the particles must avoid each other via hydrodynamics interactions; for example, the bottom particle was somehow intercalated just prior to forming the arch.

These results show the first proof-of-principle experiment for controlling and monitoring the formation of an occlusion with single particle dynamics resolution. 


\section{Probabilistic model}

Following these observations, we may infer a model for the probability of a plug formation. ${ }^{21}$ Guided by the work of Roussel et $a l^{22}$ we consider that $n \sim W / d$ particles participate in the arch bridging the two side walls to initiate the occlusion, where $W$ and $d$ are the channel width and particle's diameter, respectively. In general, $n$ may be slightly larger than $W / d$ (the arch is not straight), but this has no consequence in our semi-quantitative approach. Let us assume next that a first particle sticks to the wall with a probability $p_{0}$ and arrests for a duration $\theta$. A second particle may then encounter the first particle within the time interval $\theta$, with a probability $p_{\theta}$, and remains static for a time $\theta$ (by adhesion) to start building the arch. A third particle may collide the second particle, and so forth until the arch of $n$ particles has bridged the walls. The probability of clogging then follows $p=p_{0} p_{\theta}^{n-1}$.

A discrete reasoning may then be used to express $p_{\theta}$. On a single ballistic path in the suspension's flow, let us consider that particles occupy discrete sites of size $d$. If the volume fraction of particles has its close pack value, $\phi=\phi^{*}$, all sites are occupied. For $\phi \leq \phi^{*}$, the probability of not finding a particle in $m$ consecutive sites is $\left(1-\phi / \phi^{*}\right)^{m}$. In order to participate in the formation of the arch, the next particle moving at a velocity $V$ must be within the distance $V \theta$ upstream of the growing arch. On the aforementioned ballistic path leading to the arch, we thus find that $p_{\theta}=1-\left(1-\phi / \phi^{*}\right)^{m}$, with $m=V \theta / d$, is the probability that a particle will reach an attached particle within the time $\theta$.

Finally, the probability of clogging may be expressed as:

$p \approx p_{0}\left[1-\left(1-\phi / \phi^{*}\right)^{V_{\theta / d}}\right]^{W / d-1}$.

We show in Figure $3 c$ the variation of the probability $p$ as a function of the volume fraction $\phi$, for various values of $V \theta / d$, and for a set width of four particle diameters, $W=4 d$. When $\phi \rightarrow \phi^{*}$ (or when $W \rightarrow d$, that is a particle perfectly fitting in the channel), the probability of plugging reduces to $p=p_{0}$. In that case, only an adhesive interaction between a particle and the wall can effectively arrest the flow (our model assumes perfectly smooth walls). As $\phi$ increases, the probability increases with a steeper slope for smaller values of $V \theta / d$. This reflects the fact that, if the adhesion is short ( $\theta$ small), the volume fraction must be close to $\phi^{*}$ in order to see coordinated dynamics towards the formation of an occlusion.

Overall, our simple probabilistic model captures some intuitive understanding of a plug formation, while highlighting the significant role of adhesion in driving this process. Note that in our model, the characteristics of these adhesive interactions are contained in the parameters $p_{0}$ and $\theta$.

\section{Discussion and conclusions}

We have demonstrated a basic design of microfluidic channel, which can induce the formation of a multipleparticle clog. We showed that our device can be used to characterise the individual and collective particle dynamics leading to occlusive crises. We also presented a simple model for the probability of occlusion, which emphasises the role of adhesion in this process.

At this early stage of the study, both the experimental mimic of microvascular flow and the probabilistic model rely on significant approximations. Hence, our experimental model uses undeformable and spherical particles, in a two-dimensional flow bounded by stiff walls. Yet, we anticipate that general mechanisms of vaso-occlusion can be extracted from this simple model, and possibly extended to more realistic systems. Hence, the deformability (or lack thereof, in blood affected by disease) of the cells could potentially be accounted for by tuning the effective particle diameter $d$ in our model. Indeed, this parameter solely captures steric hindrance between particles in our theory.

In addition to the aforementioned departures from an accurate description of blood, our theoretical model makes a number of approximations. For example, it assumes that the particle velocity is constant across the channel (and equal to the mean flow velocity). But particles near the wall are driven at a lower pace than the ones near the channel centre, and attached particles locally change the flow profile. As this may have significant consequences, we are currently working on incorporating the non-uniform flow profile into our calculations. Our immediate work also focuses on obtaining an experimental measure of the probability of occlusion.

Our model also assumes the two parameters $p_{0}$ (probability of adhesion) and $\theta$ (duration of the adhesion). Adhesion is found to be central to the formation of a plug, ${ }^{23}$ and our group is currently devising an experimental technique to measure adhesive property of a microscopic object (including cells) sliding against a substrate (including endothelium). We expect such measurements will allow us to independently evaluate $p_{0}$ and $\theta$, as well as their eventual dependence with the particle velocity. Our physical approach to model occlusions, although in its first steps, will need to be reconciled against (patho)physiological characteristics of the human microcirculation. The eye microvasculature, in particular, shares similar anatomical and physiological characteristics with other end organs (eg, brain and 
kidney), and can now be visualised easily and non-invasively. ${ }^{3,24}$

The Cambridge Ophthalmological Symposium has further highlighted new methods for observing the microcirculation of the eye. For example, the 'haemoglobin video imaging' technique, presented by Paul AR Meyer, allows the measurements of blood flow in the conjunctiva and episclera, with a single blood cell resolution. Such observations, made on patients with sickle cell disease, may indeed be used to quantitatively compare our in vitro model with the actual in vivo process of vaso-occlusion.

\section{Conflict of interest}

The authors declare no conflict of interest.

\section{Acknowledgements}

This work is supported by the Isaac Newton Trust.

\section{References}

1 Secomb TW. Blood flow in the microcirculation. Annu Rev Fluid Mech 2017; 49: 443-461.

2 Popel AS, Johnson PC. Microcirculation and hemorheology. Annu Rev Fluid Mech 2005; 37: 43-69.

3 Cheung CYl, Ikram MK, Sabanayagam C, Wong TY. Retinal microvasculature as a model to study the manifestations of hypertension. Hypertension 2012; 60: 1094-1103.

4 Keane PA, Sadda SR. Retinal imaging in the twenty-first century. Ophthalmology 2014; 121: 2489-2500.

5 Campbell JP, Zhang M, Hwang TS, Bailey ST, Wilson DJ, Jia $Y$ et al. Detailed vascular anatomy of the human retina by projection-resolved optical coherence tomography angiography. Sci Rep 2017; 7: 42201.

6 Barabino GA, Platt MO, Kaul DK. Sickle cell biomechanics. Annu Rev Biomed Eng 2010; 12: 345-367.

7 Nagpal KC, Goldberg MF, Rabb MF. Ocular manifestations of sickle hemoglobinopathies. Surv Ophthalmol 1977; 21: 391-411.

8 Elagouz M, Jyothi S, Gupta B, Sivaprasad S. Sickle cell disease and the eye: old and new concepts. Surv Ophthalmol 2010; 55: 359-377.
9 Chui TYP, Mo S, Krawitz B, Menon NR, Choudhury N, Gan A et al. Human retinal microvascular imaging using adaptive optics scanning light ophthalmoscopy. Int J Retin Vitr 2016; 2: 11 .

10 Higgins JM, Eddington DT, Bhatia SN, Mahadevan L. Statistical dynamics of flowing red blood cells by morphological image processing. PLoS Comput Biol 2009; 5: e1000288.

11 Takahashi T. Microcirculation in Fractal Branching Networks. Springer: Tokyo, 2014.

12 Omori T, Imai Y, Kikuchi K, Ishikawa T, Yamaguchi T. Hemodynamics in the microcirculation and in microfluidics. Ann Biomed Eng 2014; 43: 238-257.

13 Wong KHK, Chan JM, Kamm RD, Tien J. Microfluidic models of vascular functions. Annu Rev Biomed Eng 2012; 14: 205-230.

14 Wyss HM, Blair DL, Morris JF, Stone HA, Weitz DA. Mechanism for clogging of microchannels. Phys Rev E 2006; 74: 061402.

15 Shevkoplyas SS, Yoshida T, Gifford SC, Bitensky MW. Direct measurement of the impact of impaired erythrocyte deformability on microvascular network perfusion in a microfluidic device. Lab Chip 2006; 6: 914-920.

16 Savin T, Bandi MM, Mahadevan L. Pressure-driven occlusive flow of a confined red blood cell. Soft Matter 2016; 12: $562-573$.

17 Higgins JM, Eddington DT, Bhatia SN, Mahadevan L. Sickle cell vasoocclusion and rescue in a microfluidic device. Proc Natl Acad Sci USA 2007; 104: 20496-20500.

18 Wood DK, Soriano A, Mahadevan L, Higgins JM, Bhatia SN. A biophysical indicator of vaso-occlusive risk in sickle cell disease. Sci Transl Med 2012; 4: 123ra26.

19 Cohen S, Mahadevan L. Hydrodynamics of hemostasis in sickle-cell disease. Phys Rev Lett 2013; 110: 138104.

20 To K, Lai P-Y, Pak HK. Jamming of granular flow in a two-dimensional hopper. Phys Rev Lett 2001; 86: 71-74.

21 Talbot J, Gabrielli A, Viot P. Irreversible blocking in single-file concurrent and countercurrent particulate flows. J Stat Mech 2015; 2015: P01027.

22 Roussel N, Nguyen TLH, Coussot P. General probabilistic approach to the filtration process. Phys Rev Lett 2007; 98: 114502.

23 Alapan Y, Little JA, Gurkan UA. Heterogeneous red blood cell adhesion and deformability in sickle cell disease. Sci Rep 2014; 4: 7173.

24 Wong TY, Klein R, Klein BE, Tielsch JM, Hubbard L, Nieto FJ. Retinal microvascular abnormalities and their relationship with hypertension, cardiovascular disease, and mortality. Surv Ophthalmol 2001; 46: 59-80. 